\title{
3D Human Face Identity Transfer Using Deformation Gradient
}

\author{
Tanasai Sucontphunt* \\ University of Southern California
}

\author{
Zhigang Deng ${ }^{\dagger}$ \\ University of Houston
}

\author{
Ulrich Neumann \\ University of Southern California
}

\section{INTRODUCTION}

Modeling a 3D face toward a specific person is a tedious and painstaking task even for skilled artists. Crafting a 3D cartoon-style or a $3 \mathrm{D}$ fiction-creature face to reflect a specific person likeness is even more challenging. For example, creating an ogre that keeps the actor/actress likeness or constructing a 3D avatar that reflects the person identity is an intensive process that involves high artistic skills to convey the human identity on the monster geometries. This work presents an automatic 3D face modeling system that transfers a target 3D human face identity (likeness) to any 3D character faces. This system can be used for a broad variety of a 3D face modeling such as an early stage 3D character face design or a individualized $3 \mathrm{D}$ avatars creation.

In this paper, for simplicity, we demonstrate our task by crafting a 3D monster face toward a specific human face likeness. Here, given a 3D monster model to be transformed and a 3D human face model to be transferred, our system automatically transfers the human likeness to the monster face using a deformation gradient based transferring technique.

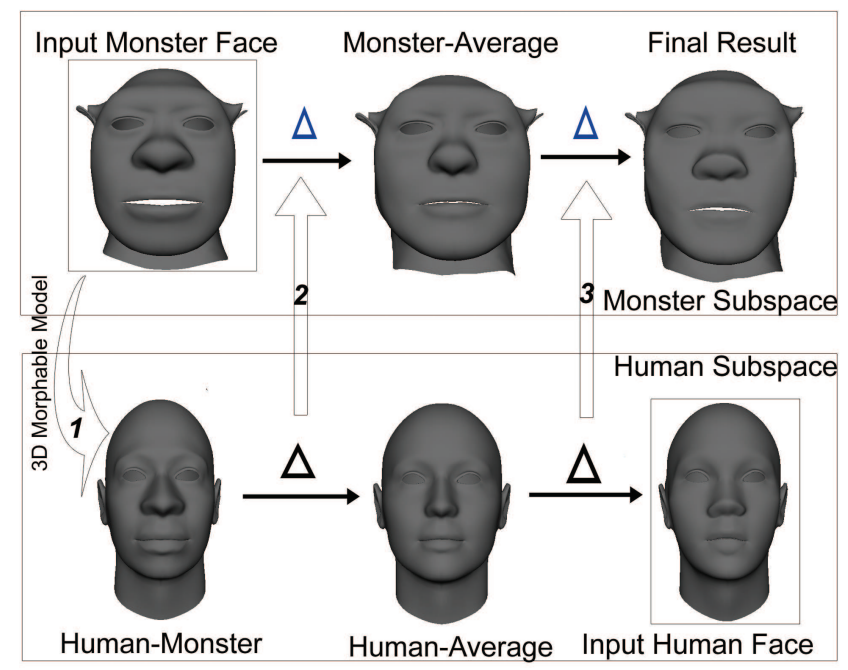

Figure 1: 3D human face identity is transferred to a 3D monster face automatically by three main steps shown as the arrows. Step-1, the input 3D monster face is morphed to a 3D human form of the monster (human-monster) by the 3D morphable model approach. Step-2, the monster-average face is constructed by the deformation gradient of the human-monster and the human-average face. This process helps remove the original monster identity. Step-3, the final result of the 3D monster face is generated from the monster-average face by the deformation gradient between the human-average face and the input 3D human face. The faces in the boxes are required inputs.

\footnotetext{
*e-mail:sucontph@usc.edu

†e-mail: zdeng@cs.uh.edu

‡e-mail: uneumann@graphics.usc.edu
}

\section{Method}

The 3D identity transfer framework is mainly composed of two procedures: an identity capturing and an identity transferring.

Identity capturing: in order to capture a target human identity, we follow a caricature modeling practice. In the caricature modeling, the caricature is crafted mainly by exaggerating the facial geometries that apparently differ from the average facial geometries. The more geometric difference from the average face, the more identity the person is revealed in the caricature. Accordingly, the difference from the human-average face can represent the human identity. This difference is the main context to transfer from the target human face to the target monster face.

Identity transferring: to correctly transfer the minute details of the geometric difference to the 3D monster model, we employ a deformation transfer framework [2]. In this technique, the geometric difference is represented by a deformation gradient. The deformation gradient is a non-translation affine transformation matrix that transforms the edges and normal vector of each triangle between two poses. Eq. 1 shows the general equation of the deformation transfer.

$$
\arg _{x} \min \|T x-S\|
$$

where $x$ is the vector containing the resultant target vertices, $T$ is a linear operator forming deformation gradient for the target model, and $S$ is the deformation gradient between two poses of the source model. In our approach, the target model is the $3 \mathrm{D}$ monster face and the source model is the 3D human face. In short, the deformation gradient between the target 3D human face and the 3D humanaverage face is the human identity to be transferred to the 3D monster face. To analytically transfer the geometries between human and monster, the human subspace and the monster subspace are constructed to arrange the models rationally. Essentially, the human with the similar identity as the monster are mapped together as a one-to-one mapping function between subspaces as shown in Fig. 1. However, if we transfer the human identity to the monster face directly, the resultant monster face will be mixed with both the transferred identity and the original monster face identity. Consequently, to neutralize the monster identity, the monster-average face is necessarily constructed first. Since the monster-average face does not exist in our dataset, the monster-average face requires to be synthesized from the available monster face. To do so, we first map the input monster face in the monster subspace to the human face in the human subspace by a 3D morphable model technique [1] forming a human-monster face. In this process, the 3D monster geometry is iteratively projected to the space spanned by human face eigen-vectors to determine the closest coefficients to re-construct the human-monster face. After that, the deformation transfer is used to transfer the difference between the human-monster face and the human-average face to the input 3D monster face to synthesize the monster-average face. Finally, with the monster-average face, the human identity is transferred to the monster-average face to generate the final target monster face.

\section{REFERENCES}

[1] V. Blanz and T. Vetter. A morphable model for the synthesis of 3D faces. In Proc. of ACM SIGGRAPH '99, pages 187-194, 1999.

[2] R. W. Sumner and J. Popović. Deformation transfer for triangle meshes. ACM Trans. Graph., 23(3):399-405, 2004. 